\title{
Key genes and pathways in measles and their interaction with environmental chemicals
}

\author{
RONGQIANG ZHANG ${ }^{1 *}$, HUALIN JIANG ${ }^{2,3^{*}}$, FENGYING LI $^{4}$, NING SU $^{5}$, \\ YI DING $^{6}$, XIANG MAO $^{7}$, DAN REN $^{2}$ and JING WANG ${ }^{1}$ \\ ${ }^{1}$ Department of Epidemiology and Health Statistics, School of Public Health, Shaanxi University of Chinese Medicine, \\ Xi'an, Shaanxi 712046; ${ }^{2}$ Department of Nursing, Health Science Center, Xi'an Jiaotong University, Xi'an, Shaanxi 710061; \\ ${ }^{3}$ Department of Medical Education, Xijing Hospital, Fourth Military Medical University, Xi'an, Shaanxi 710032; \\ ${ }^{4}$ Immunization Department of Center for Disease Control and Prevention of Xianyang, Xianyang, Shaanxi 712046; \\ Departments of ${ }^{5}$ Radiation Oncology and ${ }^{6}$ Pharmacy, Xijing Hospital, Fourth Military Medical University, \\ Xi'an, Shaanxi 710032, P.R. China; ${ }^{7}$ GoDaddy Inc, Sunnyvale, CA 95131, USA
}

Received November 2, 2017; Accepted February 2, 2018

DOI: $10.3892 /$ etm.2018.6050

\begin{abstract}
The aim of the present study was to explore key genes that may have a role in the pathology of measles virus infection and to clarify the interaction networks between environmental factors and differentially expressed genes (DEGs). After screening the database of the Gene Expression Omnibus of the National Center for Biotechnology Information, the dataset GSE5808 was downloaded and analyzed. A global normalization method was performed to minimize data inconsistencies and heterogeneity. DEGs during different stages of measles virus infection were explored using $\mathrm{R}$ software (v3.4.0). Gene Ontology and Kyoto Encyclopedia of Genes and Genomes (KEGG) pathway analysis of the DEGs were performed using Cytoscape 3.4.0 software. A protein-protein interaction (PPI) network of the DEGs was obtained from the STRING database v9.05. A total of 43 DEGs were obtained from four analyzed sample groups, including 10 highly expressed genes and 33 genes with decreased expression. The most enriched pathways based on KEGG analysis were fatty acid elongation, cytokine-cytokine receptor interaction and RNA degradation. The genes mentioned in the PPI network were mainly associated with protein binding and chemokine activity. A total of 219 chemicals were identified that may, jointly or on their own, interact with the 6 DEGs between the
\end{abstract}

Correspondence to: Dr Rongqiang Zhang, Department of Epidemiology and Health Statistics, School of Public Health, Shaanxi University of Chinese Medicine, Xi'an Avenue, Xi'an, Shaanxi 712046, P.R. China

E-mail: zhangrqxianyang@163.com

*Contributed equally

Key words: differentially expressed genes, environmental risk factors, and measles control group and patients with measles (at hospital entry), including benzo(a)pyrene (BaP) and tetrachlorodibenzodioxin (TCDD). In conclusion, the present study revealed that chemokines and environmental chemicals, e.g. BaP and TCDD, may affect the development of measles.

\section{Introduction}

Measles is a highly contagious infectious disease caused by the measles virus (MV), which spreads easily through coughs and sneezes from infected individuals. Although the measles vaccine has made an important contribution to the prevention and control of measles, there is still a long way to go to eliminate the disease (1). In 2014, an estimated 114,900 measles-associated deaths were reported across the globe, with most of these deaths occurring in children aged $<5$ years (2). In developed countries, deaths caused by measles account for $0.1-0.2 \%$ of the population; however, measles-associated mortality may be as high as $10 \%$ in low-income areas with a lack of adequate healthcare (3-5). According to the Chinese Health Statistics Yearbook, in 2013, the incidence rate of measles was 0.46 per 100,000 Chinese citizens, with a fatality rate of $0.13 \%(6-9)$. Therefore, a further reduction of the morbidity and mortality associated with measles is required. However, the complex pathogenesis of measles and the factors affecting it at the genetic, biochemical and biomechanical level, have remained to be fully elucidated.

With the continuous development of genomics technologies, high-throughput microarray and sequencing technology has also been applied to study gene expression in measles infections. Microarray analysis has also been increasingly used to explore disease-associated genes and pathways that may uncover the molecular mechanisms of measles infection (10). The GSE5808 microarray dataset was used to analyze the association between differentially expressed genes (DEGs) in different phases of measles infection and MV-induced immunological changes in a previous study, which suggested that certain genes may exhibit an extensive downregulation 
of signaling and transcriptional pathways associated with immunoregulation during the rash and convalescent phases of measles (10). These studies suggested that genes involved in immune activation and cytokine responses may have key roles in measles infection. However, the exact biological functions of these genes in the development of measles and whether these genes may be used as candidates for measles prevention and treatment, requires more detailed analysis. However, measles is the result of interactions between genes and environmental factors. The knowledge on the interaction between environmental factors and DEGs obtained from genome-wide gene expression profiling studies is currently limited.

The present study used microarray data and comprehensive bioinformatics analyses to identify DEGs between healthy controls and measles patient samples at hospital entry. Furthermore, a functional enrichment analysis for DEGs, a functional module analysis of the protein-protein interaction (PPI) network and a change trend profile analysis of the DEGs in different stages of measles infection was performed. With these comprehensive analyses, the present study aimed to identify key genes that may have roles in the development of measles virus infection and to clarify the interaction networks between environmental factors and DEGs, which may provide further information to better elucidate the mechanisms of measles and potential molecular targets for measles diagnosis and treatment.

\section{Materials and methods}

Data sources. The gene expression profile dataset GSE5808 deposited by Zilliox et al (10) was downloaded from the Gene Expression Omnibus (GEO) database of the National Center of Biotechnology Information (NCBI) (http://www.ncbi.nlm. nih.gov/geo/). The data were produced by an Affymetrix Human Genome U133A Array (HG-U133A; Affymetrix; Thermo Fisher Scientific, Inc., Waltham, MA, USA) based on the GPL96 platform, which is an efficient and robust tool for assessing gene expression changes across the human genome. This dataset was subjected to a Bioinformatics analysis in the present study. The microarray included $>1,000,000$ unique oligonucleotide features covering $>39,000$ transcript variants, which in turn represent $>33,000$ of the best characterized human genes. The GSE5808 dataset included data from 3 healthy pediatric control subjects and from 5 pediatric patients hospitalized with measles at the University Teaching Hospital in Lusaka (Lusaka, Zambia) with samples taken at hospital entry, hospital discharge, and 1-month follow-up. The demographic characteristics of the subjects are presented in Table I.

The mRNA from peripheral blood mononuclear cells (PBMCs) was used for detection. All samples were normalized with quantile normalization and summarized using robust multi-array analysis. The information on the 22,283 genes in GSE5808 was employed for bioinformatics analysis in the present study.

Data pre-processing and differential expression analysis. All the raw expression data from GSE5808 was preprocessed using R software (www.bioconductor.org) and normalization was conducted using the robust multiarray average algorithm (RMA). RMA is an algorithm used to create an expression matrix from Affymetrix data. The raw intensity values are background corrected, $\log 2$ transformed and quantile normalized. A linear model is then fitted to the normalized data to obtain an expression measure for each probe set on each array (http://www.molmine.com/magma/ loading/rma.htm). To explore the DEGs between patients with measles upon hospital admission, at discharge and one month after discharge, respectively, and the control group, the Student's t-test method implemented in the Linear Models for Microarray Data package (http://www.bioconductor .org/packages/release/bioc/html/limma.html) in R (v.3.4.0) (https://www.r-project.org/) and gene-cloud of biotechnology information (GCBI; https:/www.gcbi.com.cn/gclib/ html/index.) were utilized to calculate the significant P-values of the DEGs. The cut-off criteria for DEG screening were selected as follows: $\mathrm{P}<0.05$, false discovery rate $(\mathrm{FDR})<0.05$ and the absolute value of fold-change $(F C) \geq 2$. Subsequently, the DEGs were further identified based on the gene symbols from the original gene expression profile data.

Identification environmental chemicals affecting DEGs associated with measles. Environmental chemicals that may interact with measles-associated DEGs were accessed from the Comparative Toxicogenomics Database (CTD; http://ctdbase.org/), which provides information about interactions between chemicals and gene products and their association with diseases.

On 31 January 2017, the CTD content contained $>30.5$ million toxicogenomic connections. First, significant DEGs between the controls and patients with measles at hospital entry were defined by $\mathrm{P}<0.05, \mathrm{FDR}<0.05$ and the absolute value of $F C \geq 2$. Subsequently, environmental chemicals which interacted with measles-associated DEGs were identified from the CTD database.

Data analysis. Changes in the peripheral blood transcriptional profiles of patients with measles at different stages were analyzed using R and GCBI. To further understand the molecular functions and biological processes of the DEGs in the blood of patients with measles, Gene Ontology (GO) enrichment analysis and protein-protein interaction (PPI) analysis were performed to identify Kyoto Encyclopedia of Genes and Genomes (KEGG) pathways and an interaction network for different stages of measles infection. GO terms included three categories: Biological process, cellular component and molecular function. Fisher's exact test (two-sided) or the $\chi^{2}$ test was performed to classify the GO and KEGG pathway categories. The FDR (indicated by the Q-value) was used for P-value correction. Significantly enriched GO terms and KEGG pathways ( $\mathrm{P} \leq 0.05$; number of enriched genes, $\geq 2$ ) were identified. STRING 10.5 (http://string-db.org/), a database of known and predicted protein interactions that covers $>9.6$ million proteins from $\geq 2,000$ organisms, was also used in the analyses. The protein interaction reliability score was 0.700 (high confidence).

\section{Results}

Data pre-processing. The standardized expression profiling data after pre-processing are presented in Fig. 1. The volatility of data after normalization was greatly reduced and the 
Table I. Demographics of the cohort subjected to microarray analysis.

\begin{tabular}{lc}
\hline Group/age (months) & Sex \\
\hline Measles & \\
21 & $\mathrm{M}$ \\
79 & $\mathrm{~F}$ \\
9 & $\mathrm{M}$ \\
11 & $\mathrm{~F}$ \\
8 & $\mathrm{M}$ \\
Control & \\
26 & $\mathrm{~F}$ \\
15 & $\mathrm{M}$ \\
9 & \\
\hline
\end{tabular}

M, male; F, female.

medians of the expression values of all genes are on the same line, which provided more reliable data and information for the subsequent analyses.

Identification of DEGs in different stages of measles. A total of 43 DEGs were obtained from the groups (patients with measles vs. healthy controls), including 10 upregulated genes and 33 downregulated genes. The heat map in Fig. 2 depicts the DEGs in the different groups determined by clustering analysis. The heat map of the DEGs at different stages of measles infection and the healthy controls indicated that, compared with the controls, 33 genes had a decreased expression when the patients entered hospital, reached a minimum value at discharge and then increased, while 10 genes exhibited the opposite trend.

GCBI was used to explore and examine the temporal expression pattern of all significant DEGs to identify other trends in measles-associated gene expression. Each profile contained clusters of multiple genes that had similar temporal expression patterns. The analysis identified that the 43 DEGs were involved in a total of 26 clusters (Fig. 3). Series test cluster (STC) (11) indicated that the $P$-values (The P-value is $\mathrm{p}\left(\mathrm{X} \geq \mathrm{t}\left(\mathrm{m}_{\mathrm{i}}\right)\right), \mathrm{X} \sim \operatorname{Bin}(|\mathrm{G}|$, $\left.E_{i} /|G|\right)$ and is the significant level of single model profile. $t\left(m_{i}\right)$ is the number of genes in the $\mathrm{m}_{\mathrm{i}}$ th model profile. $\mid \mathrm{Gl}$ and $\mathrm{E}_{\mathrm{i}} / \mathrm{Gl}$ are parameters of binomial distribution which the number of genes in the model profile should obey.) (11) of clusters 3, 21 and 5 were all $<0.05$, which meant that those clusters exhibited most potential gene expression trends of patients with measles at different stages. Clusters 3 and 5 were comprised of genes that were downregulated in measles. Cluster 21 was comprised of genes that were clearly upregulated in patients with measles when they were admitted to hospital and discharged. The gene speckle-type POZ protein (SPOP) is included in profiles 3 and 5, indicating that SPOP is co-expressed in these two profiles.

GO enrichment analysis. The top five enriched terms in molecular function and biological process and two enriched terms in cellular component are listed in Table II.
The results demonstrated that the DEGs (patients with measles vs. Control) exhibited many functions, including protein binding, chemokine activity, HMG box domain binding, peptide disulfide oxidoreductase activity and type III transforming growth factor beta receptor binding. Biological process were predominantly invovled in the positive regulation of signal transduction, cellular component movement, embryonic hemopoiesis, lens development in camera-type eye and response to activity. DEGs were primarily enriched in two cellular sites: The nucleus and the cytoplasm.

KEGG enrichment analysis. The most enriched KEGG pathways were fatty acid elongation, cytokine-cytokine receptor interaction and RNA degradation. The three enriched KEGG pathways are listed in Table III.

PPI network analysis. PPI network analysis was performed using the encoded proteins from the 43 DEGs based on the STRING 9.05 database of known and predicted protein interactions. The results are presented in Fig. 4.

STRING interaction network analysis was performed for the 43 identified DEGs in the four groups and 28 encoded proteins were identified. Subsequently, the PPI network was widened by adding 20 partner proteins (CCR1, CCR5, C-C motif chemokine receptor; CUL3, Cullin 3; LSM2, LSM3, LSM4, LSM5, LSM6 and LSM7, LSMs homolog mRNA degradation associated; MAP3K5, mitogen-activated protein kinase kinase kinase 5; NDUFA12, Ubiquinone oxidoreductase subunit A12; NDUFB9, Ubiquinone oxidoreductase subunit B9; NDUFC2, Ubiquinone oxidoreductase subunit C2; PATL1, PAT1 homolog 1, processing body mRNA decay factor; RNPS1, RNA binding protein with serine rich domain 1; TGFB1, transforming growth factor beta 1; TGFB3, transforming growth factor beta 3 ; TGFBR1, transforming growth factor beta receptor 1; TXNIP, thioredoxin interacting protein; TXNRD1, thioredoxin reductase 1) with similar physiological functions to each other. A PPI with a confidence of $\geq 0.7$ and degree of interaction of nodes of $>5$ was considered as the key central node in the network in Fig. 4. The genes in the PPI network were mainly associated with chemokine activity and protein binding.

Identification of chemicals that interact with DEGs. A total of 7 DEGs $(\mathrm{Q}<0.05 ; \mathrm{P}<0.05 ; \mathrm{FC}>2)$ between the control group and patients with measles at the time-point of hospital entry are displayed in Table IV (as GTPase, IMAP family members 1-5 were detected detected as a whole in a probe, no detailed analysis of these genes was performed (Six genes in Table IV were analyzed in detail). These 6 DEGs all exhibited reduced expression compared with the control group, as indicated in Fig. 5.

A total of 219 chemicals were identified that may either singly or jointly interact with the 6 DEGs between control group and patients with measles on admission. Chemicals that may affect the expression of $>3$ DEGs are listed in Table $V$. The results indicated that benzo(a)pyrene (BaP) and tetrachlorodibenzodioxin (TCDD) interact with the expression of 6 DEGs (Ankyrin repeat domain 46, Major histocompatibility complex class II, DP $\beta 1$, Speckle-type POZ protein, Kruppel-like factor 12, Zinc finger and BTB domain containing 18 , Transforming 


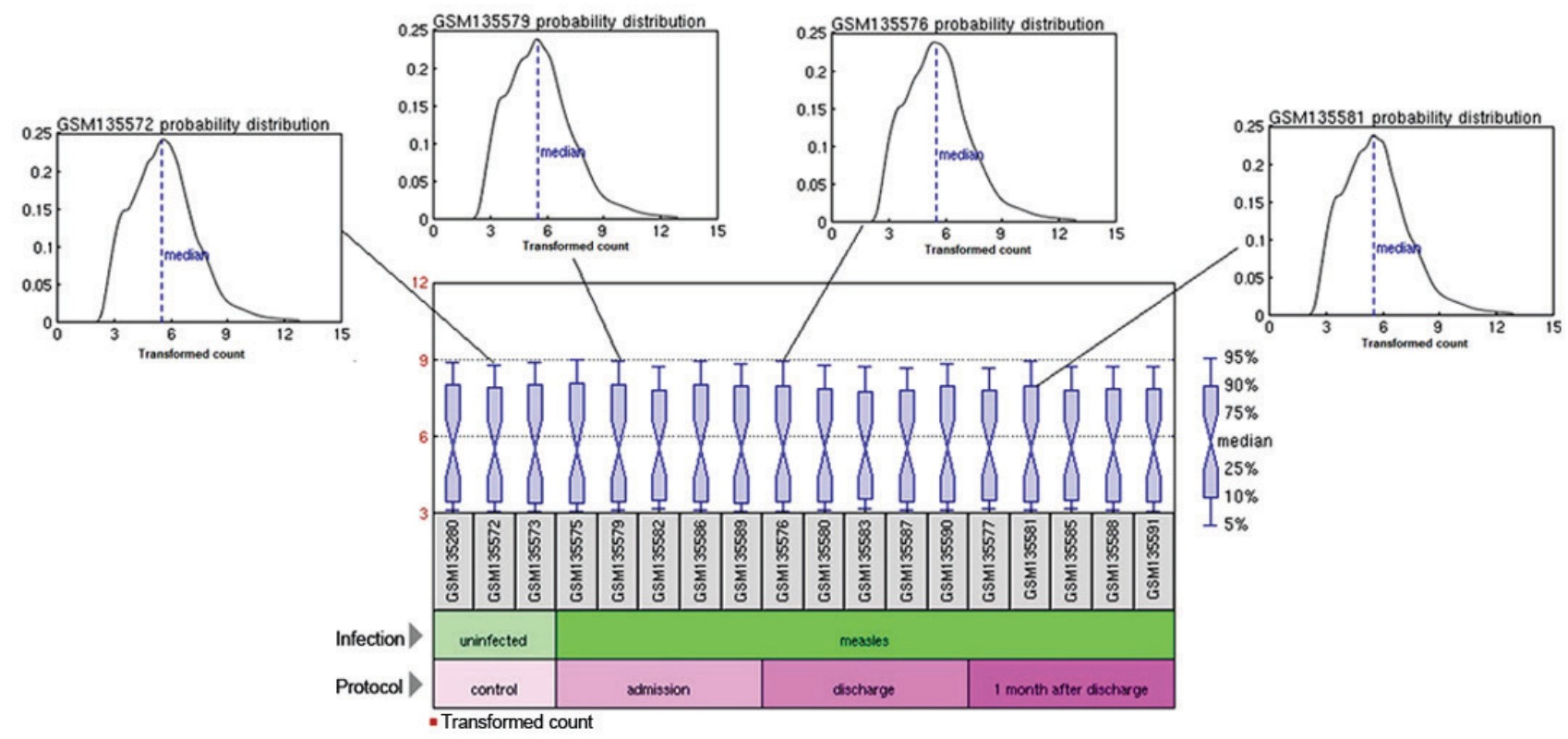

Figure 1. Distribution features of the expression data after normalization of all samples. The volatility of the data after normalization was greatly reduced and the median of expression values of all the genes are on the same line, which provided more reliable data and information for subsequent analyses.

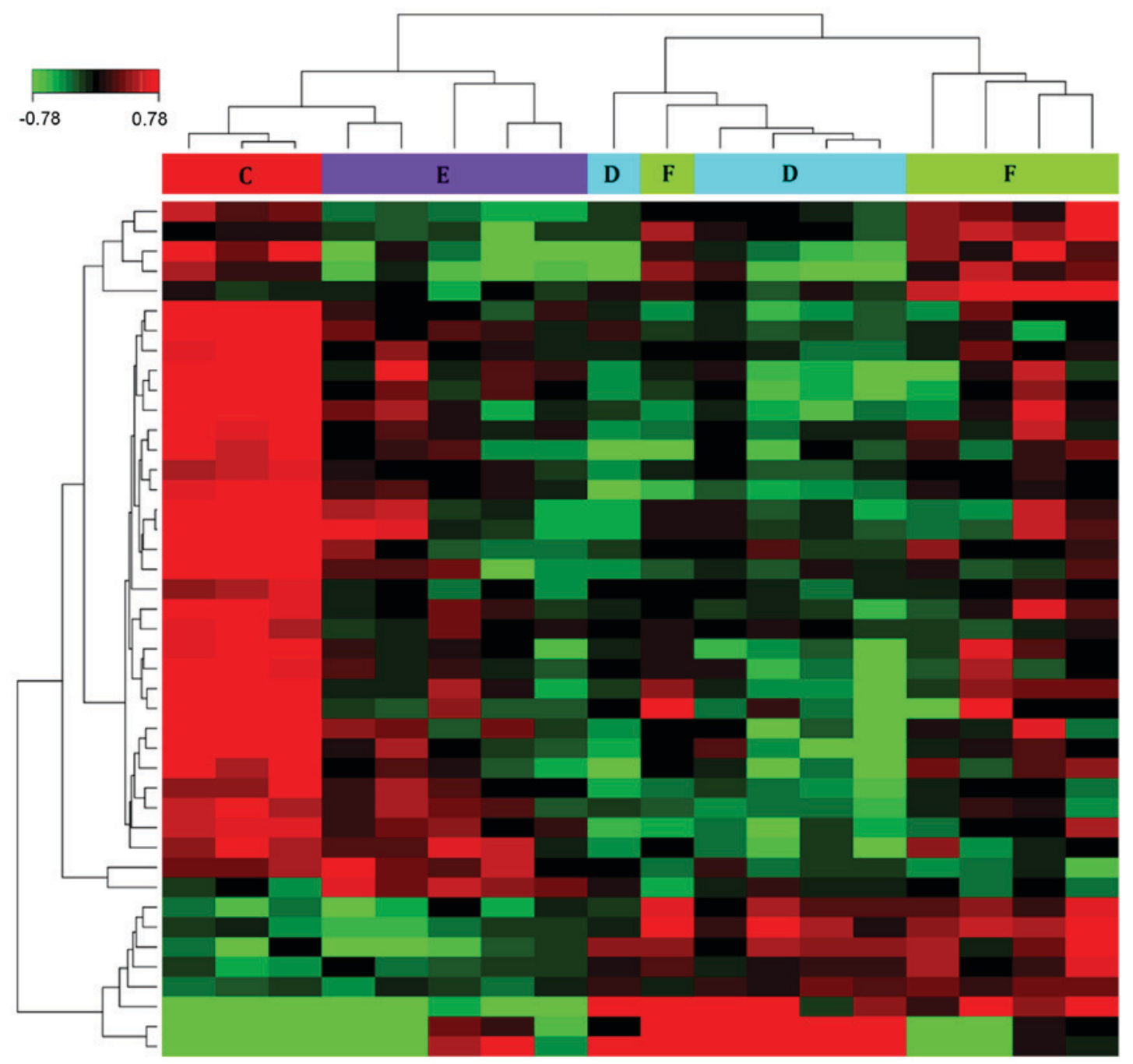

Figure 2. Heat map of differentially expressed genes between peripheral blood mononuclear cell samples of measles-infected and control subjects (C, Control; E, at hospital entry; D, at discharge; F, 1 month following discharge). Compared with the controls, 33 genes exhibited a decreased expression when patients entered hospital, reached a minimum value at discharge and then increased, while 10 genes displayed the opposite trend. Green represents low expression and red high expression. Each row represents a single gene and each column represents a sample. 
Profile 3, p-value: $3.388 \mathrm{e}-13$

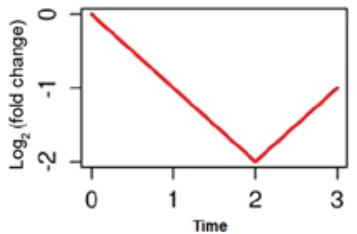

Profile 10, p-value: 0.2844

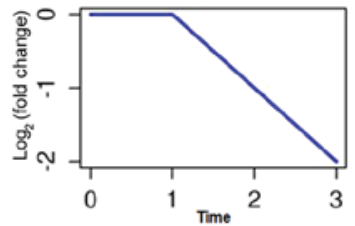

Profile 18, p-value: 0.8008

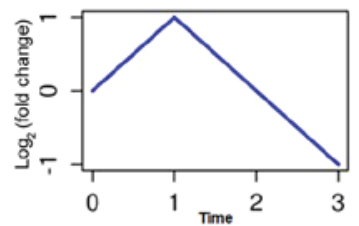

Profile 4, p-value: 1

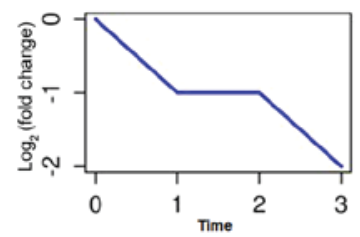

Profile 12, p-value: 1

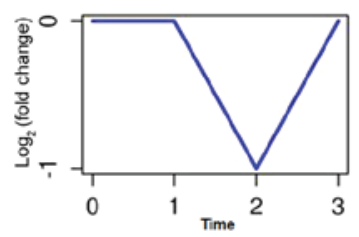

Profile 16, p-value: 1

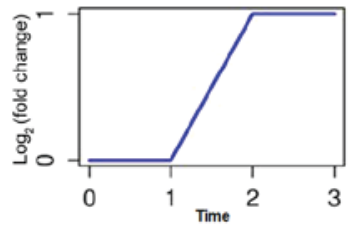

Profile 25, p-value: 1

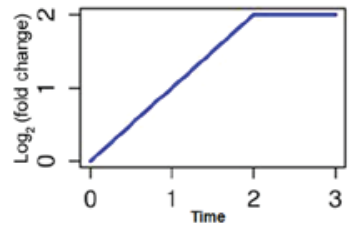

Profile 21, p-value: 0.009438

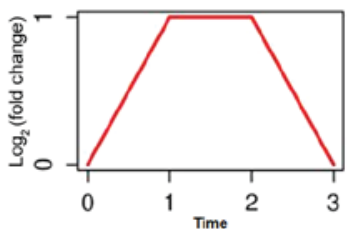

Profile 11, p-value: 0.3567

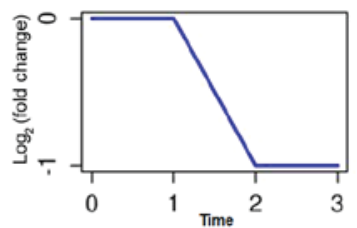

Profile 6, p-value: 0.8464

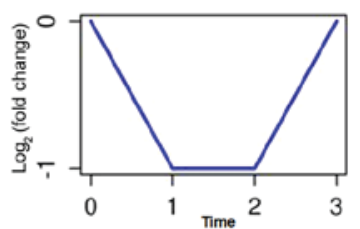

Profile 7, p-value: 1

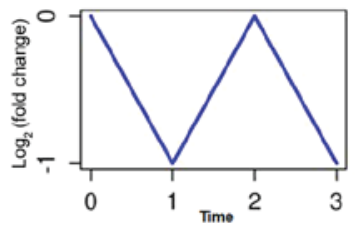

Profile 13, p-value: 1

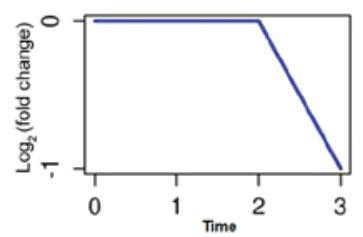

Profile 20, p-value: 1

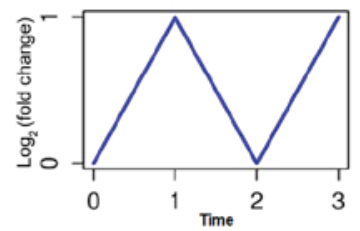

Profile 26, p-value: 1

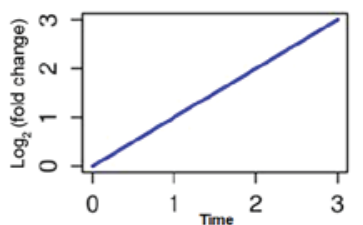

Profile 5, p-value: 0.01041

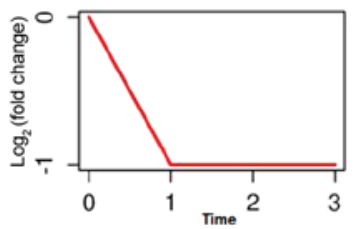

Profile 24, p-value: 0.4732

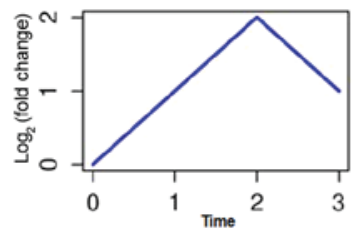

Profile 19, p-value: 0.9981

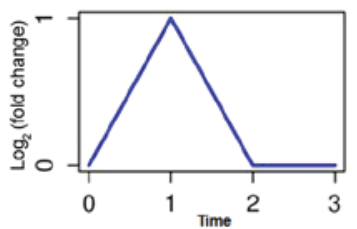

Profile 8, p-value: 1

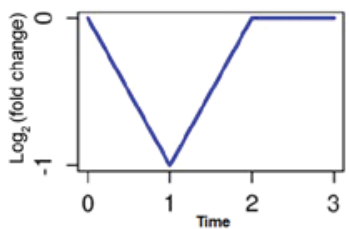

Profile 14, p-value: 1

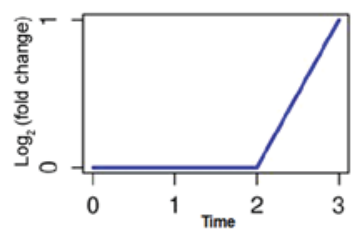

Profile 22, p-value: 1

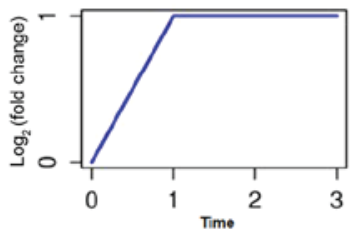

Profile 2, p-value: 0.1434

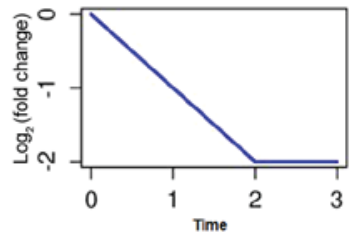

Profile 17, p-value: 0.4893

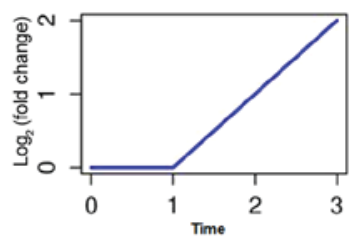

Profile 1, p-value: 1

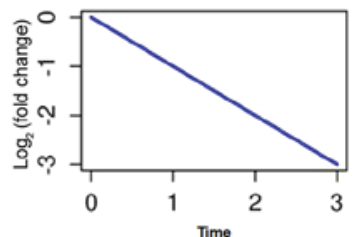

Profile 9, p-value: 1

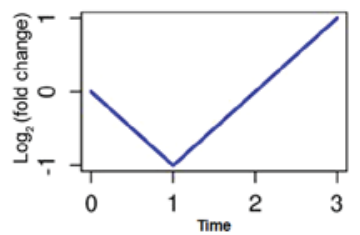

Profile 15, p-value: 1

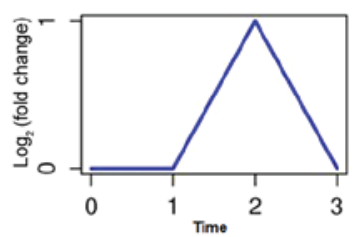

Profile 23, p-value: 1

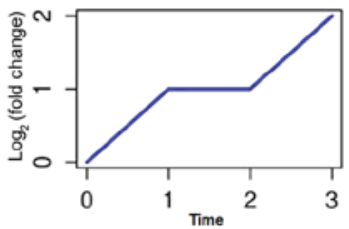

Figure 3. Profiles containing a cluster of multiple genes that have similar expression patterns were identified by gene-cloud of biotechnology information ( 0 , control; 1, admission; 2, discharge; 3, 1 month after discharge). Of these clusters, profiles 3, 5 and 21 are statistically significant (The three Ps refer to profiles 3,5 and $21<0.05$ ). Clusters 3 and 5 were comprised of genes that were downregulated in patients with measles, but when the patients were discharged, the genes in cluster 3 had the lowest expression. Cluster 21 was comprised of genes that were clearly upregulated in patients with measles when they were admitted to hospital and discharged. The gene SPOP is included in profiles 3 and 5, indicating that SPOP is co-expressed in these profiles. SPOP, speckle-type POZ protein. 
Table II. Enrichment terms in molecular function, biological process and cellular component.

\begin{tabular}{|c|c|c|c|c|}
\hline GO ontologies & GO name & P-value & FDR & Gene number \\
\hline \multirow[t]{5}{*}{ Molecular function } & Protein binding & 0.0000 & 0.0006 & 16 \\
\hline & Chemokine activity & 0.0016 & 0.0331 & 2 \\
\hline & HMG box domain binding & 0.0003 & 0.0121 & 2 \\
\hline & Peptide disulfide oxidoreductase activity & 0.0018 & 0.0331 & 1 \\
\hline & Type III transforming growth factor beta receptor binding & 0.0018 & 0.0331 & 1 \\
\hline \multirow[t]{2}{*}{ Cellular component } & Nucleus & 0.0000 & 0.0014 & 15 \\
\hline & Cytoplasm & 0.0001 & 0.0030 & 13 \\
\hline \multirow[t]{5}{*}{ Biological process } & Positive regulation of signal transduction & 0.0001 & 0.0209 & 2 \\
\hline & Cellular component movement & 0.0002 & 0.0209 & 3 \\
\hline & Embryonic hemopoiesis & 0.0003 & 0.0209 & 2 \\
\hline & Lens development in camera-type eye & 0.0005 & 0.0209 & 2 \\
\hline & Response to activity & 0.0005 & 0.0209 & 2 \\
\hline
\end{tabular}

Terms in molecular function and biological process were only presented as the top 5 .

Table III. Enriched KEGG pathways.

\begin{tabular}{|c|c|c|c|c|c|}
\hline Pathway ID & Pathway name & $\begin{array}{l}\text { Number of DEGs } \\
\text { in pathway }\end{array}$ & P-value & FDR & Genes \\
\hline 62 & Fatty acid elongation & 2 & 0.0003851 & 0.015018644 & ACOT7, HADH \\
\hline 4060 & $\begin{array}{l}\text { Cytokine-cytokine receptor } \\
\text { interaction }\end{array}$ & 3 & 0.0034654 & 0.049204993 & CCL4, TGFBR2, CCL20 \\
\hline 3018 & RNA degradation & 2 & 0.003785 & 0.049204993 & TOB2, LSM1 \\
\hline
\end{tabular}

KEGG, Kyoto Encyclopedia of Genes and Genomes; FDR, false discovery rate; ACOT7, acyl-CoA thioesterase 7; HADH, hydroxyacyl-CoA dehydrogenase; CCL4, C-C motif chemokine ligand 4; TGFBR2, Transforming growth factor $\beta$ receptor II; CCL20, C-C motif chemokine ligand 20; TOB2, transducer of ERBB2; LSM1, LSM1 homolog, mRNA degradation.

growth factor and $\beta$ receptor II), and these DEGs may have an important role in the development of measles infection.

\section{Discussion}

Measles, caused by the MV, is an acute infection that mainly affects children. Measles is a highly contagious disease and despite the availability of an effective vaccine, measles was estimated to be a direct cause of 114,900 mortalities in 2014 (2). At present, measles has become a significant public health issue worldwide. Although the burden of measles has decreased significantly during the last few decades due to the success of global measles control programs, there is still a long way to go before the goal of measles eradication is achieved. At present, a number of studies have suggested that specific gene expression signatures are produced during pathogen infection in a variety of tissues (12-14). Zilliox et al (10) reported that gene expression patterns during measles infection reflect immune responses, and that extensive downregulation of signaling and transcription pathways may be associated with these immune responses. However, specific gene expression signatures in the human genome and the mechanism of the immune response in measles infection have remained to be fully elucidated.
Studies on the molecular regulation of measles infection have made marked progress in recent years. To better characterize $\mathrm{MV}$-induced immunological changes at the transcriptional level, microarrays have been used to survey global mRNA levels in PBMCs during acute measles infection and convalescence (11). In order to obtain more valuable information, state-of-the-art bioinformatics analysis techniques were employed in the present study to re-analyze these massive gene expression datasets. A total of 43 DEGs were identified in samples from patients with measles (at hospital entry and discharge) compared with the control samples, including 10 upregulated genes and 33 downregulated genes, which suggests that in the acute phase of measles, the gene expression profiles of the patients had changed and may have been associated with the body's immune status.

Previous clinical studies suggest that at different stages of the disease, the disease characteristics have different manifestations, including gene transcription (15-17). In the present study, 26 clusters with different gene expression patterns at different stages of measles infection were identified by GCBI and profiles 3,5 and 21 were statistically significant. Profiles 3 and 5 comprised genes that were downregulated in patients with measles infection, but when the patients were discharged, 
Table IV. Six DEGs between control group and patients with measles when they entered hospital.

\begin{tabular}{|c|c|c|c|c|}
\hline Gene abbreviation & Gene definition & Fold-change & P-value & FDR \\
\hline ANKRD46 & Ankyrin repeat domain 46 & -2.435009 & 0.0001 & 0.0000 \\
\hline HLA-DPB 1 & Major histocompatibility complex, class II, DP $\beta 1$ & -2.157143 & 0.0001 & 0.0000 \\
\hline SPOP & Speckle-type POZ protein & -2.013933 & 0.0002 & 0.0411 \\
\hline KLF12 & Kruppel-like factor 12 & -2.164959 & 0.0002 & 0.0411 \\
\hline ZBTB18 & Zinc finger and BTB domain containing 18 & -2.319983 & 0.0002 & 0.0411 \\
\hline TGFBR2 & Transforming growth factor, $\beta$ receptor II & -2.465444 & 0.0003 & 0.0411 \\
\hline
\end{tabular}

FDR, false discovery rate.

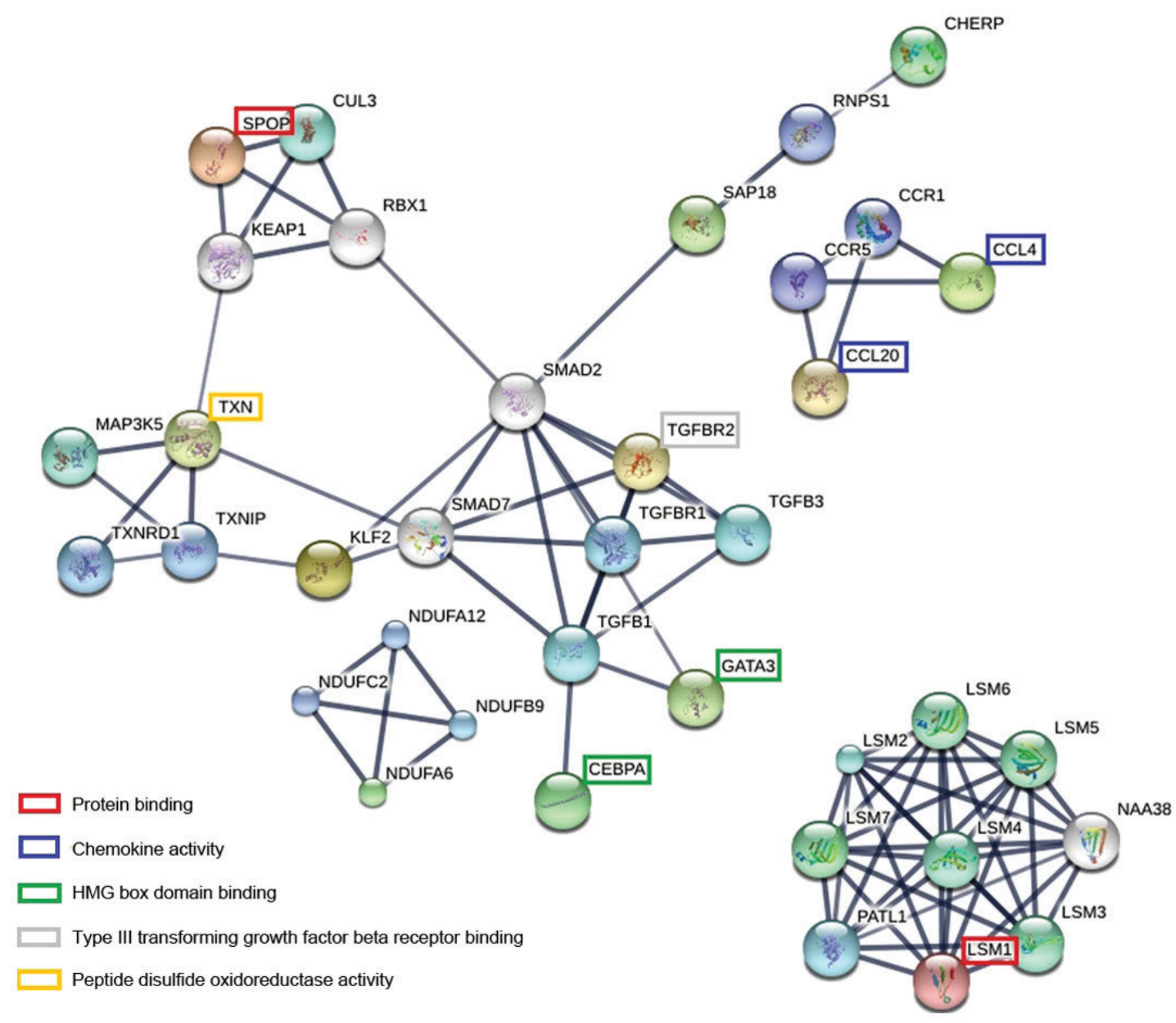

Figure 4. PPI network (confidence, $\geq 0.70$; PPI enrichment $\mathrm{P}=4.28 \times 10^{-11}$ ) of 38 coding proteins of the 43 DEGs generated with STRING. The network was expanded by including 20 additional partner proteins (CCR1 and CCR5, C-C motif chemokine receptor; CUL3, Cullin 3; LSM2, LSM3, LSM4, LSM5, LSM6 and LSM7, LSMs homolog, mRNA degradation associated; MAP3K5, mitogen-activated protein kinase kinase kinase 5; NDUFA12, Ubiquinone oxidoreductase subunit A12; NDUFB9, Ubiquinone oxidoreductase subunit B9; NDUFC2, Ubiquinone oxidoreductase subunit C2; PATL1, PAT1 homolog 1, processing body mRNA decay factor; RNPS1, RNA binding protein with serine rich domain 1; TGFB1, transforming growth factor beta 1; TGFB3, transforming growth factor beta 3; TGFBR1, transforming growth factor beta receptor 1; TXNIP, thioredoxin interacting protein; TXNRD1, thioredoxin reductase 1) that share similar physiological functions to the included DEGs. The genes with names are mainly associated with protein binding and chemokine activity. PPI, Protein-protein interaction; DEG, Differentially expressed gene. SPOP, Speckle-type POZ protein.

the genes in profile 3 had the lowest expression levels. Cluster 21 comprised genes that were clearly upregulated in patients with measles when they were admitted to hospital and discharged. The SPOP gene is included in profiles 3 and 5 , indicating that SPOP is involved in the two profiles. SPOP was first reported as a mutated gene in human prostate cancer and its substrates are implicated in several essential cellular functions, including apoptosis, secondary messenger formation and transcriptional repression (18-20). The results of the present analysis indicated that SPOP expression in PBMCs from patients with measles was significantly lower than that in healthy controls. 
Table V. Identified differently expressed environmental response genes in the blood samples of patients with measles (upon hospital entry) and healthy controls.

Environmental response DEGs between measles and controls

ANKRD46, HLA-DPB1, KLF12, SPOP, TGFBR2, ZBTB18

ANKRD46, HLA-DPB1, KLF12, TGFBR2, ZBTB18

ANKRD46, KLF12, SPOP, TGFBR2, ZBTB18

ANKRD46, HLA-DPB1, SPOP, TGFBR2

ANKRD46, KLF12, SPOP, TGFBR2

HLA-DPB1, KLF12, SPOP, ZBTB18

ANKRD46, HLA-DPB1, TGFBR2

ANKRD46, SPOP, TGFBR2

ANKRD46, KLF12, TGFBR2
Chemicals

\section{Benzo(a)pyrene}

Tetrachlorodibenzodioxin

Valproic acid

Cyclosporine

Pirinixic acid

Tetradecanoylphorbol acetate

Acetaminophen

Ionomycin

Bisphenol A

Antirheumatic agents

Cadmium chloride

Diuron

Copper sulfate

Nanotubes, carbon

Phenobarbital

Estradiol

2,4,5,2',4',5'-hexachlorobiphenyl

Methyl methanesulfonate

Potassium chromate(VI)

Isotretinoin

Dexamethasone

Carbamazepine

$\mathrm{N}, \mathrm{N}, \mathrm{N}^{\prime}, \mathrm{N}^{\prime}$-tetrakis(2-pyridylmethyl)ethylenediamine Methotrexate

N-Methyl-3,4-methylenedioxyamphetamine

Oxygen

7,8-Dihydro-7,8-dihydroxybenzo(a)pyrene 9,10-oxide

Dextroamphetamine

Trichostatin A

Decitabine

Cobaltous chloride

Dietary fats

9,10-Dimethyl-1,2-benzanthracene

vinclozolin

Ethinyl estradiol

C646 compound

Tretinoin

Arsenic trioxide

Propiconazole

Quercetin

Vancomycin

Diethylnitrosamine

Carbon tetrachloride

Vanadates

Tobacco smoke pollution

Lipopolysaccharides

Isoproterenol

Gentamicins

Ammonium chloride

8-Bromo cyclic adenosine monophosphate 
Table V. Continued.

Environmental response DEGs between measles and controls

Chemicals

KLF12, SPOP, ZBTB18

\author{
Dibutyl phthalate \\ Oxaliplatin
}

DEGs, differentially expression genes; ANKRD46, ankyrin repeat domain 46; HLA-DPB1, major histocompatibility complex class II; DP $\beta 1$; SPOP, speckle-type POZ protein; KLF12, kruppel-like factor 12; ZBTB18, zinc finger and BTB domain containing 18; TGFBR2, transforming growth factor $\beta$ receptor II.

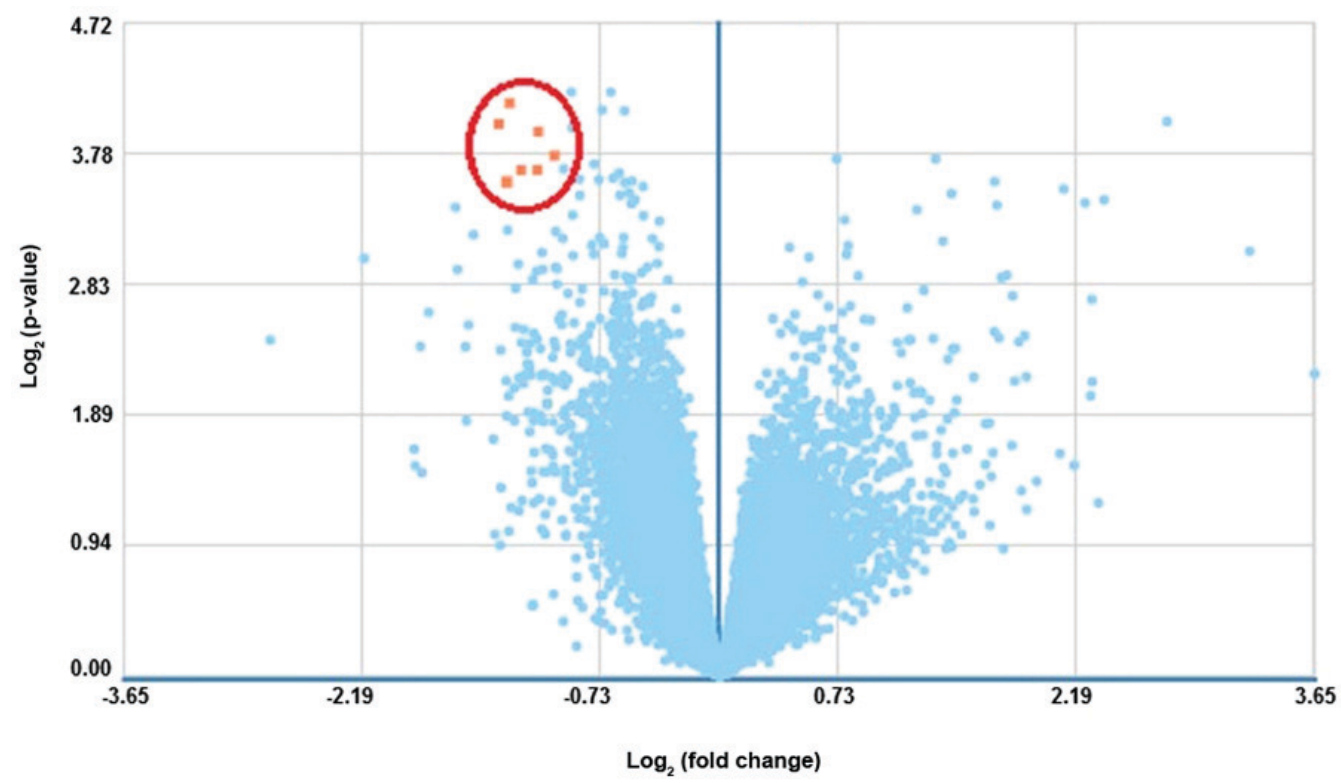

Figure 5. Volcano plot of the differentially expressed genes (orange squares in the circle) between control group and patients with measles when they entered hospital (GTPase IMAP family members 1-5 included).

Chemokines are a large family of proteins $(6-14 \mathrm{kDa})$ that mediate a wide range of biological activities, particularly in the normal immune system (21). Chemokines are essential for immune system architecture and development, and immune surveillance, which are mediated by the chemokine signaling pathway (22). Chemokines activate leukocytes and stimulate various effector functions of these cells (23). The results of the present study indicated that the chemokine signaling pathway may have an important role in MV-induced immunological changes, which may help to explain, at least partly, the prolonged alteration of lymphocyte responses that are characteristic of measles.

$\mathrm{BaP}$ is a member of the benzopyrenes, which are formed by the fusion of a benzene ring to pyrene. BaP is considered to be important due to its toxicity and abundance in the environment, and has been reported to induce immune suppression, phototoxicity, as well as neurological and reproductive dysfunction in in vivo experiments (24-26). However, to the best of our knowledge, no previous study has reported on the association between $\mathrm{BaP}$ and infectious diseases. However, the immune suppression induced by $\mathrm{BaP}$ in animals or humans may affect the development of infectious diseases. For instance, BaP may inhibit white blood cells from differentiating into macrophages, which may affect the first line of defense to fight infections in the body (27). The molecular mechanisms of the effects of $\mathrm{BaP}$ on the immune system have remained elusive. The results of the present study indicated that the 6 DEGs between patients with measles and healthy controls are all able to interact with $\mathrm{BaP}$, which suggests that upon MV infection of the body, BaP may serve a role in the occurrence and development of measles. TCDDs, also called dioxins, are environmental, highly lipophilic and persistent pollutants usually emitted from combustion facilities. TCDDs may contaminate the food chain and accumulate in adipose tissues, and the diet is considered one of the major sources of exposure to TCDD for the general population (28). TCDDs are known to be endocrine-disrupting chemicals and are suspected to increase the risk of cancer, including breast cancer (29-31). To the best of our knowledge, no epidemiological or basic research studies have reported an association between TCDD or dioxin exposure and measles infection. The present results indicated that TCDD also interacts with 6 DEGs between patients with measles and healthy controls, and these interactions may affect the immune system directly or indirectly (for instance, they may first disrupt the normal biological activities through the chemokine pathway), which may increase the risk of measles development. 
In conclusion, the present study performed detailed bioinformatics analyses of a dataset from the NCBI's GEO database to identify key biomarkers and their interactions with environmental chemicals in the development of measles. The genome-wide analysis included differential expression analysis, GO enrichment analysis, KEGG analysis and PPI analysis, as well as an analysis of the interaction of the DEGs with environmental chemicals. The present results may provide insight into the genetic basis of measles infection and the possible role of environmental chemicals in the development of measles. Further functional studies are required to validate the results of the present study and further elucidate the mechanisms of measles infection.

\section{Acknowledgements}

Not applicable.

\section{Funding}

This study was funded by Youth Research Project of Shaanxi University of Chinese Medicine (grant number: 2015NQ05).

\section{Availability of data and materials}

The analyzed data sets generated during the study are available from the corresponding author on reasonable request.

\section{Authors' contributions}

RZ and FL planned the study. RZ, FL, HJ, JW, NS, YD, XM, and DR performed the data analysis, wrote and modified the manuscript. RZ and HJ submitted the study.

\section{Ethical approval and consent to participate}

Not applicable.

\section{Consent for publication}

Not applicable.

\section{Competing interests}

The authors declare that they have no competing interests.

\section{References}

1. World Health Organization. Measles Fact Sheets, 2016 http://www.who.int/mediacentre/factsheets/fs286/en/. Accessed September 29, 2017.

2. Centers for Disease Control and Prevention (CDC): Measles-United States, 2011. MMWR Morb Mortal Wkly. Rep 61: 253-257, 2012.

3. World Health Organization: Measles-Rubella Bulletin, 2017. http://iris.wpro.who.int/bitstream/handle/10665.1/13539/Meas les-Rubella-Bulletin-2017-Vol-11-No-01.pdf?ua=1. Accessed September 29, 2017.

4. No authors listed: Measles vaccines: WHO position paper. Wkly Epidemiol Rec 84: 349-360, 2009 (In English, French).

5. Zheng J, Zhou Y, Wang $\mathrm{H}$ and Liang X: The role of the China experts advisory committee on immunization program. Vaccine 28 (Suppl 1): A84-A87, 2010.
6. Zhang RQ,Li HB,Li FY,Han LX and Xiong YM: Epidemiological characteristics of measles from 2000 to2014: Results of a measles catch-up vaccination campaign in Xianyang, China. J Infect Public Health 10: 624-629, 2017

7. Li L, Yu WZ, Shui TJ, Ma C, Wen N and Liang XF: Analysis on epidemiological characteristics of age distribution of measles in China during 2003-2006. Chin J Vaccines Immun 13: 101-105, 2007.

8. Wang Z, Yan R, He H, Li Q, Chen G, Yang S and Chen E: Difficulties in eliminating measles and controlling rubella and mumps: A Cross-sectional study of a first measles and rubella vaccination and a second measles, mumps, and rubella vaccination. PLoS One 9: e89361, 2014.

9. Liu Y, Lu P, Hu Y, Wang Z, Deng X, Ma F, Tao H, Jia C, Ding X, Yang $\mathrm{H}$, et al: Cross-sectional surveys of measles antibodies in the Jiangsu Province of China from 2008 to 2010: The effect of high coverage with two doses of measles vaccine among children. PLoS One 8: e66771, 2013.

10. Zilliox MJ, Moss WJ and Griffin DE: Gene expression Changes in Peripheral blood mononuclear cells during measles virus infection. Clin Vaccine Immunol 14: 918-923, 2007.

11. Xiao S, Mo D, Wang Q, Jia J, Qin L, Yu X, Niu Y, Zhao X, Liu $X$ and Chen Y: Aberrant host immune response induced by highly virulent PRRSV identified by digital gene expression tag profiling. BMC Genomics 11: 544, 2010.

12. Honda M, Kaneko S, Kawai H, Shirota Y and Kobayashi K: Differential gene expression between chronic hepatitis B and C hepatic lesion. Gastroenterology 120: 955-966, 2001.

13. Sankaran S, Guadalupe M, Reay E, George MD, Flamm J, Prindiville T and Dandekar S: Gut mucosal T cell responses and gene expression correlate with protection against disease in long-term HIV-1-infected nonprogressors. Proc Natl Acad Sci USA 102: 9860-9865, 2005.

14. Yu SY, Hu YW, Liu XY, Xiong W, Zhou ZT and Yuan ZH: Gene expression profiles in peripheral blood mononuclear cells of SARS patients. World J Gastroenterol 11: 5037-5043, 2005.

15. Rota PA, Liffick SL, Rota JS, Katz RS, Redd S, Papania M and Bellini WJ: Molecular epidemiology of measles viruses in the United States, 1997-2001. Emerg Infect Dis 8: 902-908, 2002.

16. Rota PA, Featherstone DA and Bellini WJ: Molecular epidemiology of measles virus. Curr Top Microbiol Immunol 330: 129-150, 2009.

17. Riddell MA, Rota JS and Rota PA: Review of the temporal and geographical distribution of measles virus genotypes in the prevaccine and postvaccine eras. Virol J 2: 87, 2005.

18. Blattner M, Liu D, Robinson BD, Huang D, Poliakov A, Gao D, Nataraj S, Deonarine LD, Augello MA, Sailer V, et al: SPOP mutation drives prostate tumorigenesis in vivo through coordinate regulation of PI3K/mTOR and AR signaling. Cancer Cell 3: 436-451, 2017.

19. Zhang L, Peng S, Dai X, Gan W, Nie X, Wei W, Hu G and Guo J: Tumor suppressor SPOP ubiquitinates and degrades EgIN2 to compromise growth of prostate cancer cells. Cancer Lett 390: 11-20, 2017.

20. Zhi X, Tao J, Zhang L, Tao R, Ma L and Qin J: Silencing speckle-type POZ protein by promoter hypermethylation decreases cell apoptosis through upregulating Hedgehog signaling pathway in colorectal cancer. Cell Death Dis 7: e2569, 2016.

21. Rump L, Mattey DL, Kehoe O and Middleton J: An initial investigation into endothelial CC chemokine expression in the human rheumatoid synovium. Cytokine 97: 133-140, 2017.

22. Zheng Y, Han GW, Abagyan R, Wu B, Stevens RC, Cherezov V, Kufareva I and Handel TM: Structure of CC Chemokine receptor 5 with a potent chemokine antagonist reveals mechanisms of chemokine recognition and molecular mimicry by HIV. Immunity 46: 1005-1017.e5, 2017.

23. Prabhakaran S, Rizk VT, Ma Z, Cheng CH, Berglund AE, Coppola D, Khalil F, Mulé JJ and Soliman HH: Evaluation of invasive breast cancer samples using a 12-chemokine gene expression score: Correlation with clinical outcomes. Breast Cancer Res 19: 71, 2017.

24. Arfsten DP, Schaeffer DJ and Muneny DC: The effects of near ultraviolet radiation on the toxic effects of polycyclic aromatic hydrocarbons in animals and plants: A review. Ecotoxicol Environ Saf 33: 1-24, 1996.

25. Saunders CR, Ramesh A and Shockley DC: Modulation of neurotoxic behavior in F-344 rats by temporal disposition of benzo(a)pyrene. Toxicol Lett 129: 33-45, 2002. 
26. Yamaguchi K, Near R, Shneider A, Cui H, Ju ST and Sherr DH: Fluoranthecene-induced apoptosis in murine $\mathrm{T}$ cell hybridomas is independent of the aromatic hydrocarbon receptor. Toxicol Appl Pharmacol 139: 144-152, 1996.

27. Kang HG, Jeong SH, Cho $\mathrm{MH}$ and Cho JH: Changes of biomarkers with oral exposure to benzo(a)pyrene, phenanthrene and pyrene in rats. J Vet Sci 8: 361-368, 2007.

28. Fries GF: A review of the significance of animal food products as potential pathways of human exposures to dioxins. J Anim Sci 73: 1639-1650, 1995.

29. Kogevinas M: Human health effects of dioxins: Cancer, reproductive and endocrine system effects. Hum Reprod Update 7: 331-339, 2001.
30. Schug TT, Janesick A, Blumberg B and Heindel JJ: Endocrine disrupting chemicals and disease susceptibility. J Steroid Biochem Mol Biol 127: 204-215, 2011.

31. Liem AK, Fürst P and Rappe C: Exposure of populations to dioxins and related compounds. Food Addit Contam 17: 241-259, 2000 .

(i) (2) This work is licensed under a Creative Commons Attribution-NonCommercial-NoDerivatives 4.0 International (CC BY-NC-ND 4.0) License. 